\title{
Zeolite Imidazolate Frameworks-67 Precursor to Fabricate a Highly Active Cobalt-Embedded N-Doped Porous Graphitized Carbon Catalyst for the Thermal Decomposition of Ammonium Perchlorate
}

\author{
Huiyu Liu, Zhiliang Guo, Qingchun Zhang,* Bo Jin, and Rufang Peng*
}

Cite This: ACS Omega 2021, 6, 25440-25446

Read Online

ABSTRACT: The more apparent specific heat release at a lower high-temperature decomposition (HTD) temperature of ammonium perchlorate (AP) poses a challenge for the development of highly active catalysts. In this work, a well-designed cobaltembedded N-doped porous graphitized carbon (Co@NC) catalyst is obtained by high-temperature calcination of a zeolite imidazolate frameworks-67 precursor, in which the cobalt catalytic active center realizes effective nanoscale dispersion; meanwhile, the cobalt and $\mathrm{N}$-doped porous graphitized carbon can release considerable heat after oxidation, and the cobalt oxides have an excellent catalytic effect on reducing the HTD temperature of AP. The catalytic activity of Co@NC was tested by a differential thermal analytical method. The results indicated that the HTD peak of AP was

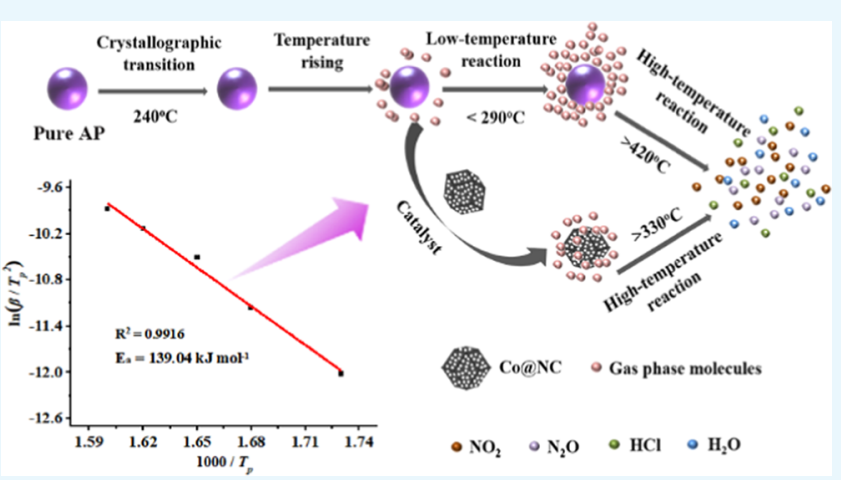
significantly decreased by $100.5^{\circ} \mathrm{C}$, the apparent activation energy of the HTD reaction of AP was reduced by $82.0 \mathrm{~kJ} \mathrm{~mol}^{-1}$, and the heat release compared with pure AP increased 2.9 times. On teh basis of these findings, Co@NC is expected to be one of the best candidate materials for AP thermal decomposition.

\section{INTRODUCTION}

Composite solid propellants (CSPs) mainly include a binder (hydroxyl-terminated polybutadiene), a metal fuel (aluminum powder), and an oxidant (ammonium perchlorate (AP)), of which AP generally accounts for more than half of the CSPs. ${ }^{1}$ Moreover, the thermal decomposition ability of AP can significantly affect the combustibility of CSPs. ${ }^{2}$ In general, lowering the high-temperature decomposition (HTD) temperature and increasing the heat release of AP can achieve an increase of the combustion rate, high specific impulse, and lowpressure exponent of CSPs. ${ }^{3,4}$ To improve the combustion performance of CSPs, the catalytic thermal decomposition of AP was extensively studied using the catalytic activities of metals, ${ }^{5,6}$ transition-metal oxides (TMOs), ${ }^{7-9}$ and composite catalysts. ${ }^{10-12}$ However, most of the work focuses only on reducing the HTD temperature ignoring the heat release of AP. Considering that the heat release of AP can also significantly influence the combustibility of CSPs, there is an urgent need to develop a catalyst that can reduce the HTD temperature and increase the heat release of AP. The combustion exothermic reaction of carbon materials (for instance, carbon nanotubes, graphene, and amorphous carbon) with an oxidizing gas produced by the thermal decomposition of AP has excellent performance in increasing heat release but exhibits mediocre performance in reducing the HTD temper- ature, ${ }^{13-16}$ except for the purposely designed ordered porous graphitized carbon with a large specific surface area. ${ }^{17}$

However, metal-organic frameworks (MOFs) with excellent stability, uniform particle size, and a large specific surface area were often used as precursors to fabricate metallic single-atom catalysts by high-temperature calcination under an inert atmosphere, in which the metal atom orderly embedded $\mathrm{N}$-doped porous graphitized carbon, ${ }^{18-20}$ which may not only realize the catalytic synergistic effect of a metallic active center and $\mathrm{N}$-doped porous graphitized carbon but also ensure catalytic stability by inhibiting migration and agglomeration.

In this work, zeolite imidazolate frameworks-67 (ZIF-67), as a cobalt-based MOF material, was used as a precursor to fabricate a cobalt-embedded $\mathrm{N}$-doped porous graphitized carbon (Co@NC) catalyst, in which the cobalt and N-doped porous graphitized carbon can be oxidized by gas produced by AP decomposition to release a large amount of heat, ${ }^{21}$ as well as the oxidation products of cobalt- $\mathrm{CoO}, \mathrm{CoCl}_{2}$, and $\mathrm{Co}_{3} \mathrm{O}_{4}$-have excellent catalytic activity for reducing the

Received: June 30, 2021

Accepted: September 9, 2021

Published: September 24, 2021 


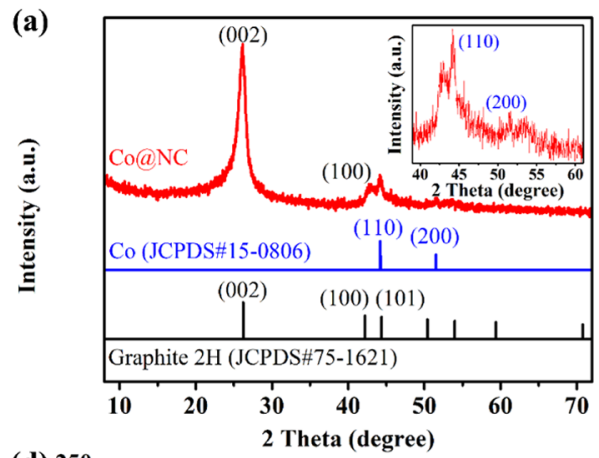

(b)
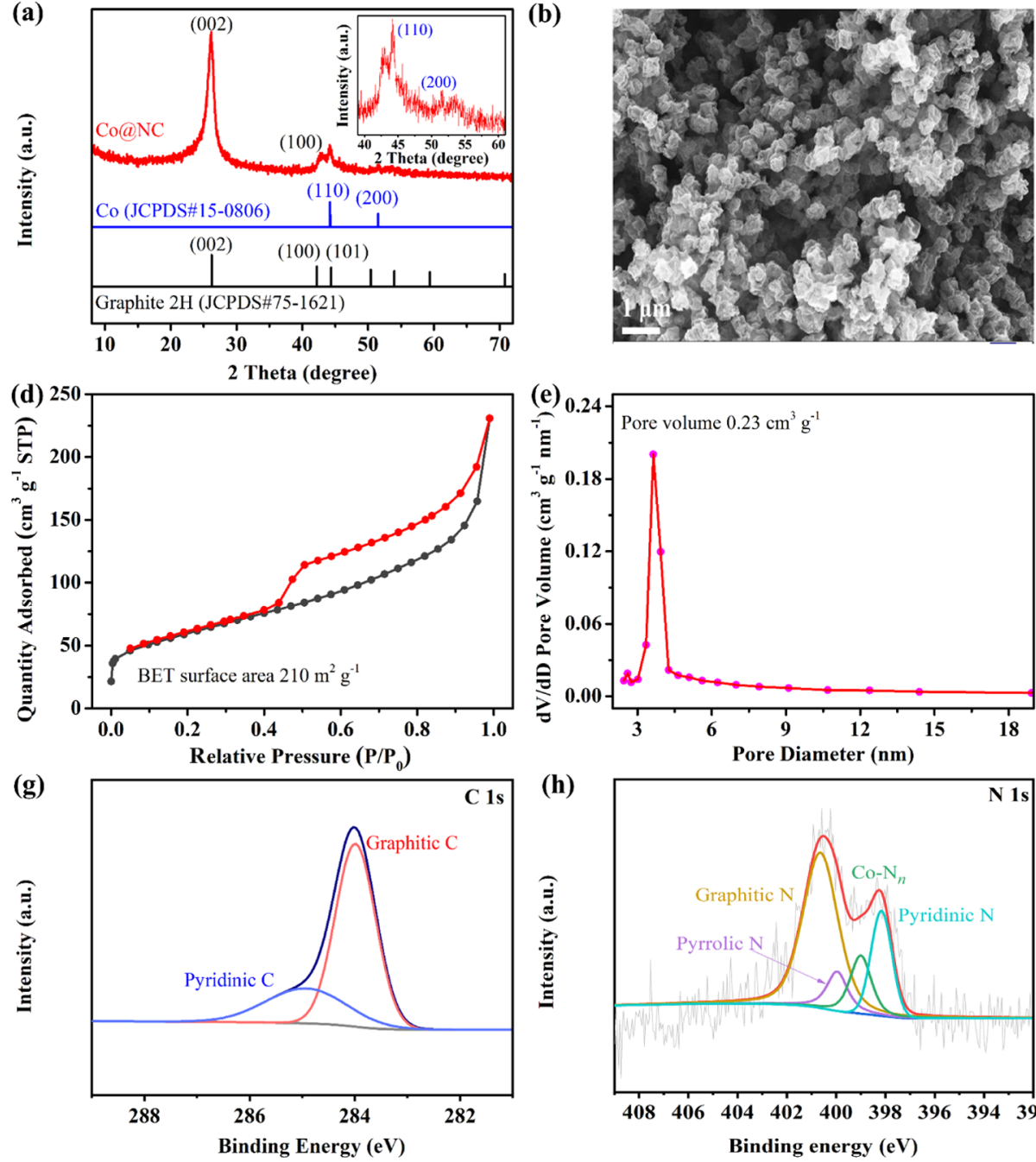

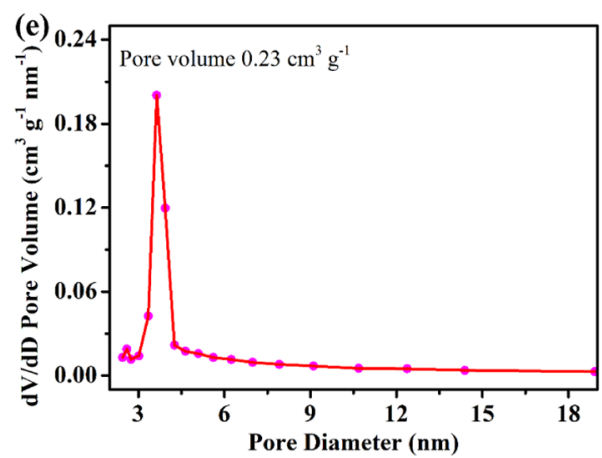

(h)

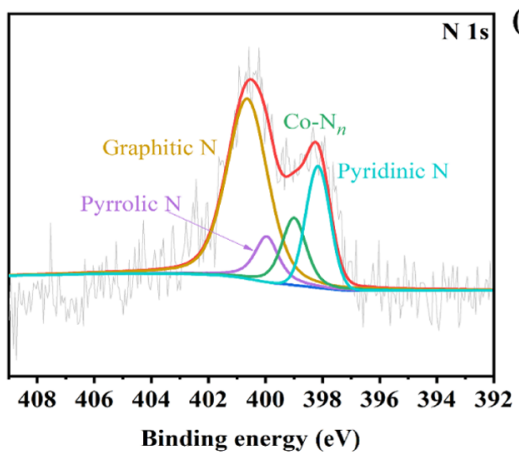

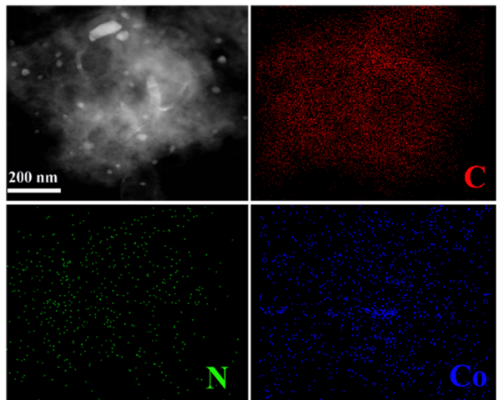
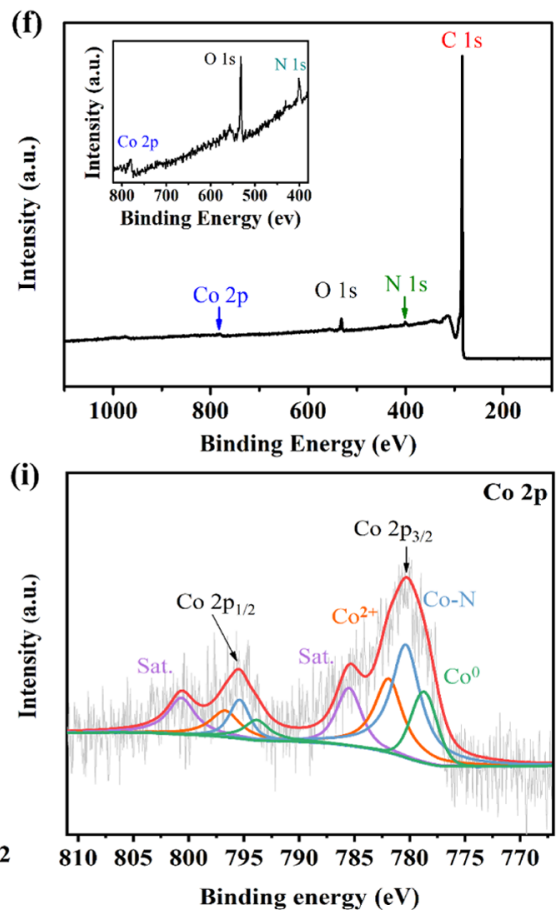

Figure 1. (a) XRD patterns, (b) SEM images, and (c)TEM-EDS elemental mapping of the Co@NC catalyst. (d) $\mathrm{N}_{2}$ adsorption-desorption isotherms and (e) pore size distributions of the Co@NC catalyst. (f) Survey scan XPS spectrum, (g) high-resolution C 1s spectrum, (h) highresolution N 1s spectrum, and (i) high-resolution Co 2p spectrum of the Co@NC catalyst.

HTD temperature. ${ }^{22,23}$ This catalyst is expected to serve as an ideal high-efficiency catalyst for the thermal decomposition of AP. To the best of our knowledge, thermocatalytic properties of ZIF-67-derived Co@NC are as yet unexplored.

\section{RESULTS AND DISCUSSION}

2.1. Characteristics of ZIF-67. The structure of the prepared ZIF-67 was characterized by an X-ray diffractometer (XRD). All of the XRD peaks of ZIF-67 are the same as the characteristic peaks obtained by the theoretical calculation and simulation, and no other hybrid peaks belonging to the SOD topology are found (Figure S1). ${ }^{24}$ The XRD result shows that the ZIF-67 material has been developed and successfully synthesized. ZIF-67 has strong and sharp characteristic diffraction peaks, indicating that the ZIF-67 powder is highly crystalline. In addition, the diffraction peak intensity of the (011) crystal plane in ZIF-67 is significantly higher than the other peaks, indicating that the ZIF-67 crystal has a good (110) orientation. $^{25}$ Fourier transform infrared (FTIR) spectroscopy is used to explore the coordination relationship between $\mathrm{Co}^{2+}$ and 2-methylimidazole (Figure S2). Due to the formation of hydrogen bonds between the pyrrole group and pyridine nitrogen, 2-methylimidazole shows wide and strong characteristic absorption peaks between 2200 and $3300 \mathrm{~cm}^{-1}$. Meanwhile, 2-methylimidazole possesses a strong characteristic absorption peak at $1840 \mathrm{~cm}^{-1}$, which corresponds to the $\mathrm{N}-\mathrm{H}$ stretching vibration. By contrast, the characteristic absorption peak of the $\mathrm{N}-\mathrm{H}$ stretching vibration disappeared from the spectra of ZIF-67, indicating that deprotonation of the ligand had occurred completely during the formation of the ZIF structure. Additionally, ZIF-67 exhibits a new characteristic absorption peak at $431 \mathrm{~cm}^{-1}$, attributed to the $\mathrm{Co}-\mathrm{N}$ stretching mode, which is consistent with previous reports. ${ }^{26}$ Therefore, FTIR spectroscopy results of ZIF-67 confirmed that the $\mathrm{Co}^{2+}$ and 2-methylimidazole coordinated in the framework.

The morphology and size of ZIF-67 were characterized by a scanning electron microscope (SEM). The SEM images show that ZIF-67 possessed very clear rhombohedral features with an average particle size of about $2 \mu \mathrm{m}$ (Figure S3). The structure diagram of ZIF-67 reveals that one cobalt atom coordinates with four nitrogen atoms to form a Co- $\mathrm{N}_{4}$ reticulation structure (Figure S4). The results of thermogravimetric analysis (TGA) show that ZIF-67 has excellent thermal stability. The TGA curve shows that ZIF-67 has no obvious weight loss during the heating process from room temperature to $450{ }^{\circ} \mathrm{C}$ until the temperature is higher than $450{ }^{\circ} \mathrm{C}$, and 

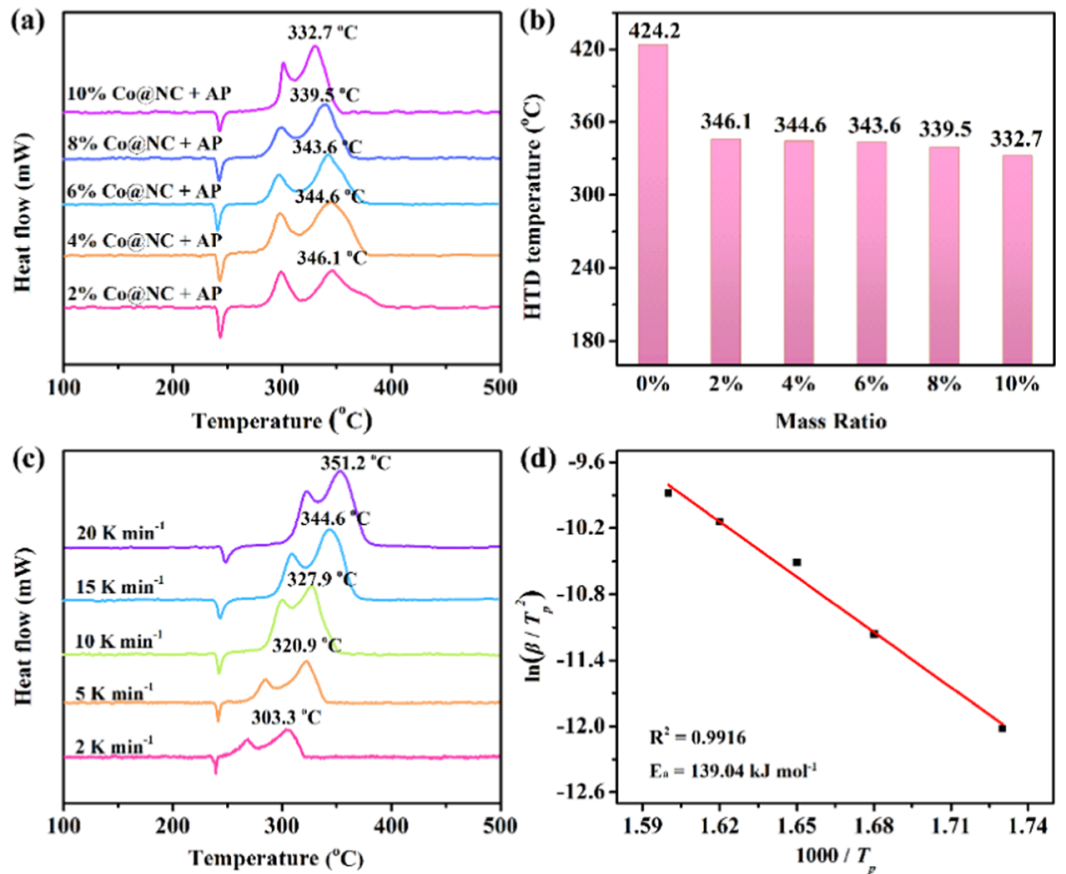

Figure 2. (a) DTA curves of AP with different contents of Co@NC, (b) HTD temperature of pure AP and Co@NC/AP mixtures, (c) DTA curves of AP with 10 wt \% Co@NC at different heating rates, and $(\mathrm{d})$ dependence of $\ln \left(\beta / T_{\mathrm{p}}^{2}\right)$ on 1000/T of the Co@NC/AP mixture.

ZIF-67 begins to lose weight quickly, which corresponds to the thermal decomposition of the 2-methylimidazole ligand (Figure S5).

2.2. Characteristics of the Co@NC Catalyst. The XRD spectra show that Co@NC has two characteristic peaks, in which the broad shoulder peak belongs to the (002) diffraction peak of graphite carbon in the range of $20-30^{\circ}$ and the sharp peak around $44^{\circ}$ corresponds to the (101) crystal plane of graphitic carbon. ${ }^{27}$ Furthermore, the material has weak characteristic diffraction peaks at about 44 and $51^{\circ}$, which match with the (111) and (200) crystal planes of the facecentered cubic crystals of cobalt nanoparticles (Figure 1a),28 which are fully enclosed by graphitic layers and cannot be eliminated even by acid pickling. The microscopic morphology and size of Co@NC were analyzed by SEM. Although Co@ NC deformed after high-temperature calcination, the frame almost remained visible and the size was almost the same as before (Figure 1b).

In addition, the elemental composition of Co@NC was verified by energy-dispersive $\mathrm{X}$-ray spectroscopy (EDS). The element distribution mapping demonstrated that cobalt was well dispersed throughout in $\mathrm{N}$-doped porous graphitized carbon (Figure 1c) and combined with the weak face-centered cubic diffraction peak in $\mathrm{XRD}$, indicated that there is no obvious aggregation of cobalt atoms. The $\mathrm{N}_{2}$ adsorptiondesorption isotherm curves were measured to determine the specific surface area and porosity of $\mathrm{Co} @ \mathrm{NC}$, which are two powerful parameters that influence the catalytic performance of samples. Interestingly, Co@NC exhibited distinctly strong adsorption at a relatively low pressure $\left(P / P_{0}<0.05\right)$, demonstrating the existence of micropores (Figure 1d). Furthermore, Co@NC exhibited a conspicuous hysteresis loop and gradual uptake in its $\mathrm{N}_{2}$ sorption isotherms at a relative pressure $>0.45$, corresponding to the type IV isotherm curve, according to the IUPAC classification (Figure 1e). ${ }^{29}$ As expected, Co@NC retains the characteristics of ZIF-67 and possesses a rather high Brunauer-Emmett-Teller (BET) specific surface area of $210 \mathrm{~m}^{2} \mathrm{~g}^{-1}$ and a Barrett-JoynerHalenda (BJH) pore volume of $0.23 \mathrm{~cm}^{3} \mathrm{~g}^{-1}$, indicating that Co@NC not only has adequately high active density centers but also provides enough porous channels for the electrolyte solution to facilitate mass transport and electron transfer.

Nevertheless, the amount of Co of the Co@NC catalyst was quantified by inductively coupled plasma-optical emission spectrometry (ICP-OES). The result displayed that the content of Co was 0.19 wt \% in the Co@NC catalyst (Table $\mathrm{S} 1$ ). X-ray photoelectron spectroscopy (XPS) was frequently used to explore the surface chemical composition and element valence state of the material. The results confirmed that Co@ NC mainly includes three kinds of elements, for instance, C, N, and Co (Figure 1f). The high-resolution energy spectrum of $\mathrm{C}$ $1 \mathrm{~s}$ shows the presence of two peaks, with he main intense pair of peaks located at 284.3 and $284.9 \mathrm{eV}$, which are matched with pure graphitic sites and pyridnic carbon, ${ }^{30}$ respectively (Figure $1 \mathrm{~g}$ ). There are four different types of $\mathrm{N}$ in the highresolution $\mathrm{N}$ 1s spectrum of Co@NC, which correspond to pyridinic $\mathrm{N}(398.2 \mathrm{eV}), \mathrm{Co}-\mathrm{N}_{n}(399.0 \mathrm{eV})$, pyrrolic $\mathrm{N}(399.9$ $\mathrm{eV}$ ), and graphitic $\mathrm{N}(400.8 \mathrm{eV}$ ) (Figure $1 \mathrm{~h}){ }^{31,32}$ Due to the spin-orbit coupling, the Co $2 \mathrm{p}$ spectrum demonstrates two sharp peaks at $781 \mathrm{eV}\left(\mathrm{Co} 2 \mathrm{p}_{3 / 2}\right)$ and $795 \mathrm{eV}\left(\mathrm{Co} 2 \mathrm{p}_{1 / 2}\right)$. The spectrum of Co $2 \mathrm{p}_{3 / 2}$ is divided into three species, and the corresponding binding energies are $778.7,780.4$, and $781.9 \mathrm{eV}$, which further proves the presence of metallic $\mathrm{Co}^{0}$ and the active site of $\mathrm{Co}-\mathrm{N}_{n}$ that exists (Figure 1i). ${ }^{33-35}$

2.3. Catalytic Activity Studies. The catalytic performance of Co@NC was studied by a differential thermal analyzer (DTA). To demonstrate the catalytic property of Co@NC on $\mathrm{AP}$ decomposition, AP and Co@NC were mixed uniformly with different weight ratios ranging from 98:2 to 90:10. The total amount of $3.0 \mathrm{mg}$ of mixed samples was used for the DTA test. As we all know, the crystallographic system of AP changes from orthogonal to cubic during heating, which is accom- 
panied by an endothermic reaction at $244{ }^{\circ} \mathrm{C}$ (Figure 2a). As the temperature continues to rise, $\mathrm{AP}$ continues to undergo thermal decomposition accompanied by two violent exothermic reactions, corresponding to low-temperature thermal decomposition (LTD) at about $300{ }^{\circ} \mathrm{C}$ and HTD at about $424{ }^{\circ} \mathrm{C}$. When the temperature rises to the maximum temperature of LTD decomposition, AP will partially decompose, primarily involving a gas-solid two-phase reaction. During the HTD process, AP mainly undergoes a gas-phase reaction, which completely converts perchloric acid and ammonia molecules into small gas molecules. ${ }^{36,37}$ With the content of Co@NC increasing, the exothermic peak of the HTD decomposition step of the mixed sample was significantly reduced from 346.1 to $332.7{ }^{\circ} \mathrm{C}$ (Figure $2 \mathrm{a}$ ). Compared with pure AP, the introduction of Co@NC will observably decrease the HTD temperature of AP decomposition (Figure 2b).

To further study the kinetic performance of Co@NC on the catalytic thermal decomposition of AP, Co@NC and AP were mixed uniformly at a mass ratio of $1: 9$, which was tested by the decomposition process of the mixed samples by DTA at different heating rates (Figure $2 \mathrm{c}$ ). The heating rate variation will cause the exothermic peak temperature distinction with the sample under the same test conditions. The faster the heating rate, the peak temperature of HTD significantly moves toward a higher temperature. Therefore, the HTD peak temperature of AP catalytic decomposition and heating rate can be used for calculating several important kinetic parameters. As we all know, there is a strong correlation between the exothermic peak temperature and the different heating rates during the AP decomposition process, which can be calculated according to the Kissinger formula ${ }^{38}$

$$
\ln \left(\frac{\beta}{T_{\mathrm{p}}^{2}}\right)=\ln \left(\frac{A R}{E_{\mathrm{a}}}\right)-\frac{E_{\mathrm{a}}}{R T_{\mathrm{p}}}
$$

where $\beta$ corresponds to the heating rate $\left(\mathrm{K} \mathrm{min}^{-1}\right), E_{\mathrm{a}}$ is the apparent activation energy $\left(\mathrm{kJ} \mathrm{mol}{ }^{-1}\right), T_{\mathrm{p}}$ is the peak temperature of $\operatorname{HTD}(\mathrm{K}), \mathrm{A}$ is a pre-exponential factor, and $\mathrm{R}$ is the ideal gas constant $\left(8.314 \mathrm{~J} \mathrm{~mol}^{-1} \mathrm{~K}^{-1}\right)$. According to the above correlation, a straight line can be described, utilizing the term $\ln \left(\beta / T_{\mathrm{p}}{ }^{2}\right)$ that varies linearly with $1000 / T_{\mathrm{p}}$, and the powerful kinetic reaction parameters are obtained from the slope of the straight line and the intercept, such as the preexponential factor and activation energy. The activation energy of pure AP approaches is about $221.0 \mathrm{~kJ} \mathrm{~mol}^{-1}$ during the HTD decomposition process. ${ }^{39}$ Nevertheless, when Co@NC was introduced into AP, the decomposition activation energy of AP was significantly decreased to $139.0 \mathrm{~kJ} \mathrm{~mol}^{-1}$ (Figure 2d). Therefore, Co@NC can effectively promote AP thermal decomposition.

The catalytic performances of Co@NC and other reported catalysts are shown in Table 1. Graphene ${ }^{13}$ and metal oxides, $^{40,41}$ especially cobalt oxides, ${ }^{22}$ exhibit excellent performance in increasing the heat release and decreasing the HTD peak of AP. By contrast, Co@NC combines the advantages of carbon materials and the metallic active center and possesses a balanced comprehensive performance.

2.4. Catalytic Mechanisms. The thermal decomposition process of AP usually involves the transformation of solidliquid-gas three-phase flow, which is very complicated. The decomposition of AP can be roughly divided into three stages. First of all, the AP crystal undergoes a phase transition from orthorhombic to cubic at about $244{ }^{\circ} \mathrm{C}$. Then, AP mainly
Table 1. Catalytic Property of Various Catalysts in the Thermal Decomposition of AP

\begin{tabular}{lccccc}
\multicolumn{1}{c}{ catalysts } & $\begin{array}{c}\text { content } \\
(\text { wt \% })\end{array}$ & $\begin{array}{c}\mathrm{HTD} \\
\text { peak } \\
\left({ }^{\circ} \mathrm{C}\right)\end{array}$ & $\begin{array}{c}\Delta T \\
\left({ }^{\circ} \mathrm{C}\right)^{a}\end{array}$ & $\begin{array}{c}\Delta E_{\mathrm{a}} \\
\left(\mathrm{kJ} \mathrm{mol}^{-1}\right)^{b}\end{array}$ & $\Delta H / \Delta H_{\mathrm{AP}}{ }^{c}$ \\
graphene $^{d}$ & 10 & 336.5 & 69.7 & & 3.4 \\
$\mathrm{Co}_{3} \mathrm{O}_{4}{ }^{c}$ & 5 & 301.8 & 152.0 & 53.0 & 1.9 \\
$\mathrm{CuO}^{f}$ & 5 & 331.6 & 90.4 & 119.9 & 1.9 \\
$\alpha-\mathrm{Fe}_{2} \mathrm{O}_{3}{ }^{g}$ & 5 & 367.6 & 54.3 & 67.9 & 1.5 \\
$\mathrm{GO} / \mathrm{TAG}^{-C \mathrm{Co}^{h}}$ & 20 & 328.0 & 77.1 & & 1.6 \\
$\mathrm{ZIF}-67^{i}$ & 5 & 335 & 89.8 & 142.7 & 2.9 \\
$\mathrm{Co} @ \mathrm{NC}^{j}$ & 10 & 327.9 & 100.5 & 82.0 & 2.9
\end{tabular}

${ }^{a} \Delta T$ is the difference of the HTD peak between pure AP and the catalyst/AP mixture. ${ }^{b} \Delta E_{\mathrm{a}}$ is the difference in the apparent activation energy between pure AP and the catalyst/AP mixture. ${ }^{c} \Delta H / \Delta H_{\mathrm{AP}}$ is the ratio of heat release of the catalyst/AP mixture compared with pure AP. ${ }^{d}$ Reference $13 .{ }^{e}$ Reference $22 .{ }^{f}$ Reference $40 .{ }^{g}$ Reference 41. ${ }^{h} \mathrm{GO} / \mathrm{TGA}-\mathrm{Co}$ is graphene oxide-doped cobalt of triaminoguanidine in ref 42. ${ }^{i}$ Reference 43. ${ }^{j}$ The $\Delta H / \Delta H_{\mathrm{AP}}$ of Co@NC was determined from the ratio of the HTD peak area of the Co@NC/AP mixture compared with pure AP according to the data in Table S2.

undergoes a gas-solid two-phase reaction in the LTD stage, generating the intermediate products $\mathrm{HClO}_{4}$ and $\mathrm{NH}_{3}$, which are adsorbed on the solid AP crystal lattice as gases. Finally, a gas-phase reaction dominates, which converts the intermediate product and AP into small gas molecules in the HTD stage. ${ }^{44}$

To further analyze the composition of the gas generated during the AP thermal decomposition process, thermal gravity analysis-Fourier transform infrared (TGA-FTIR) spectroscopy is frequently used as an analytical tool to detect AP decomposition gas products in real time. The three-dimensional (3D) FTIR spectrum of the thermal decomposition of AP with 10 wt \% Co@NC demonstrated that AP began to violently decompose between 300 and $400{ }^{\circ} \mathrm{C}$, producing a large number of gas products, and obvious strong absorption peaks appeared in the fingerprint region of the FTIR spectrum (Figure 3a), which were attributed to the LTD and HTD decomposition stages of AP. The TGA curve of the Co@NC/ $\mathrm{AP}$ mixture also revealed that the thermal decomposition process was divided into two steps, corresponding to the LTD and HTD stages, in which weight-loss rates were 14.68 and $83.88 \%$, respectively (Figure $3 \mathrm{~b}$ ), and the HTD temperature of AP decomposition was $341.9{ }^{\circ} \mathrm{C}$, corresponding to the strongest absorption peak position in the three-dimensional FTIR spectrum. To study the composition of the gas-phase products in the AP decomposition process, the FTIR spectra of several characteristic temperatures were intercepted for analysis in the temperature range of violent decomposition of AP. All of the generated gas products come from the LTD and HTD stages during the decomposition of AP. The FTIR spectra corresponding to the gas product were generated at $378{ }^{\circ} \mathrm{C}$, which could explicitly prove that the product contains $\mathrm{H}_{2} \mathrm{O}$ and $\mathrm{NH}_{3}\left(3400-3650\right.$ and $\left.1650-1620 \mathrm{~cm}^{-1}\right), \mathrm{HCl}$ (1750-3000 cm $\left.{ }^{-1}\right) ; \mathrm{N}_{2} \mathrm{O}$ and $\mathrm{NO}_{2}$ (2202-2238, 1380-1320, and $\left.840-800 \mathrm{~cm}^{-1}\right)$, and $\mathrm{ClO}_{3}\left(1000-900 \mathrm{~cm}^{-1}\right){ }^{45,46}$ In addition, the gas products detected at 348 and $408{ }^{\circ} \mathrm{C}$ were identical to those at $378{ }^{\circ} \mathrm{C}$ (Figure 3c).

Compared with pure AP, the surface of $\mathrm{Co} @ \mathrm{NC}$ underwent a series of reactions that promoted the thermal decomposition of AP. Therefore, Co@NC can not only significantly reduce the activation energy of AP during the LTD and HTD decomposition stages but also efficiently promote the 
(a)

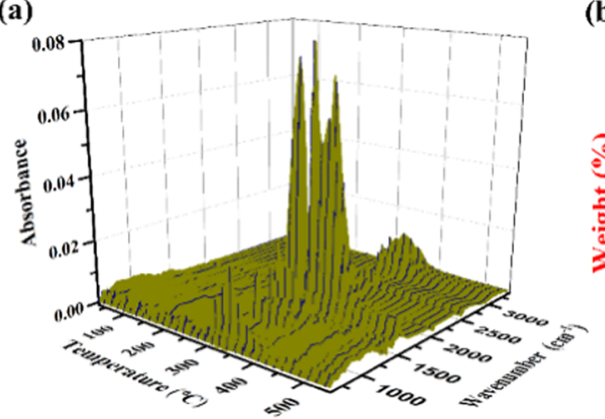

(b)

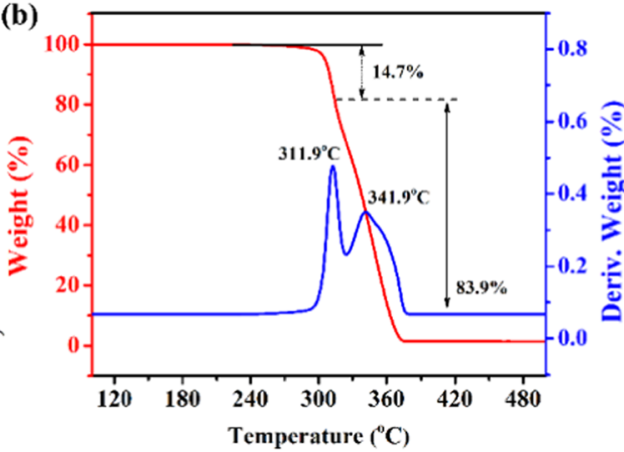

(c)

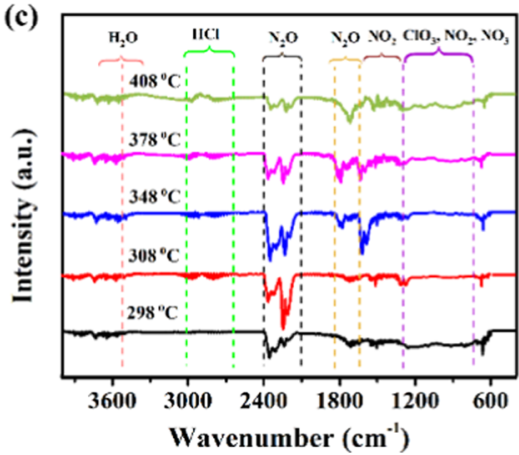

Figure 3. (a) 3D FTIR spectra of the thermal decomposition of the Co@NC/AP mixture. (b) TGA curve of the Co@NC/AP mixture. (c) FTIR spectra of gas products during the thermal decomposition of AP by the Co@NC catalyst.

reduction of $\mathrm{HClO}_{4}$ to $\mathrm{HCl}$ and the oxidation of $\mathrm{NH}_{3}$ to stable nitrogen oxide compounds. ${ }^{47,48}$ The introduction of Co@NC into AP caused the reaction decomposition temperature to drop sharply and the generated decomposition heat increased prominently.

\section{CONCLUSIONS}

In summary, Co@NC was successfully prepared by calcining the ZIF-67 precursor. The differential thermal analytical results indicated that the HTD peak was reduced by $100.5{ }^{\circ} \mathrm{C}$, the apparent activation energy of the AP decomposition reaction was reduced by $82.0 \mathrm{~kJ} \mathrm{~mol}^{-1}$, and the heat release compared with pure AP increased 2.9 times. By contrast, Co@NC combines the advantages of carbon materials and metal oxides, possesses a balanced comprehensive performance, and is expected to serve as a potential high-efficiency catalyst for the thermal decomposition of AP.

\section{EXPERIMENTAL SECTION}

4.1. Materials and Apparatus. All reagents and solvents used were of analytical grade and used without further purification. 2-Methylimidazole and $\mathrm{Co}\left(\mathrm{NO}_{3}\right)_{2} \cdot 6 \mathrm{H}_{2} \mathrm{O}$ were purchased from Aladdin Biochemical Technology Co., Ltd. (Shanghai, China). Methanol was purchased from Chengdu Colon Chemical Co., Ltd. (Chengdu, China).

The XRD patterns were tested by a PANalytical X'Pert Pro $\mathrm{X}$-ray diffractometer with monochromatized $\mathrm{Cu} \mathrm{K}_{\alpha}(\lambda=$ $0.15418 \mathrm{~nm}$, Holland). The microscopic morphologies were observed with a Zeiss Supra 55VP field emission scanning electron microscope (FE-SEM, Germany) after gold sputtering and a Zeiss Libra 200 transmission electron microscope (TEM, Germany). Elemental analysis was carried out on the extra equipment of the TEM. Differential thermal analysis was performed using a WCR-2B differential thermal analyzer (DTA, China) at a heating rate of $10{ }^{\circ} \mathrm{C} \mathrm{min}^{-1}$ from room temperature to $500{ }^{\circ} \mathrm{C}$. Thermogravimetric analysis (TGA) were performed on a TA Instruments SDT Q600 simultaneous thermal analyzer, with a temperature range from room temperature to $500{ }^{\circ} \mathrm{C}$ at a heating rate of $10{ }^{\circ} \mathrm{C} \mathrm{min}-1$ and

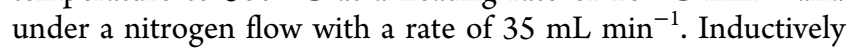
coupled plasma-optical emission spectrometry (ICP-OES Agilent 720ES) was used for the analysis of the Co content. X-ray photoelectron spectroscopy (XPS) was conducted using $\mathrm{Al} \mathrm{K} \alpha$ radiation, and spectra were collected on a scanning $\mathrm{X}$ ray microprobe (Thermo ESCALAB 250XI). The BrunauerEmmett-Teller (BET) surface areas and porous structure of the as-synthesized sample were measured using an ASAP 2460 volumetric analyzer from the $\mathrm{N}_{2}$ adsorption-desorption isotherm at $77 \mathrm{~K}$. Thermal gravity analysis-Fourier transform infrared spectroscopy (TGA-FTIR) was carried out on a TGIR-MS combined instrument (Perkin-Elmer).

4.2. Preparation of the Co@NC Catalyst. First, Co$\left(\mathrm{NO}_{3}\right)_{2} \cdot 6 \mathrm{H}_{2} \mathrm{O}(5.82 \mathrm{~g}, 20.0 \mathrm{mmol})$ and 2-methylimidazole $(6.16 \mathrm{~g}, 75.0 \mathrm{mmol})$ were separately dissolved in $150 \mathrm{~mL}$ of methanol and were then mixed and continuously stirred for 24 $\mathrm{h}$ at room temperature. The purple precipitates were collected by centrifugation and dried in a drying oven at $60{ }^{\circ} \mathrm{C}$ for $12 \mathrm{~h}$ to obtain the ZIF-67 powder. Subsequently, the ZIF-67 powder was placed in a tube furnace and heated to $1000{ }^{\circ} \mathrm{C}$ (heating rate of $5{ }^{\circ} \mathrm{C} \mathrm{min}^{-1}$ ) in a stream of Ar to obtain a black solid powder (Figure 4). To remove the oxides generated

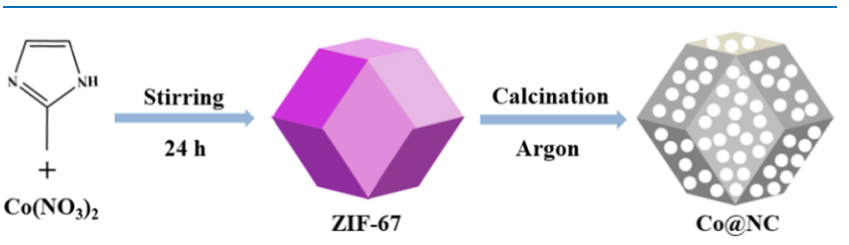

Figure 4. Preparation process of the Co@NC catalyst.

during the calcination process, the black solid powder was immersed in $0.5 \mathrm{M} \mathrm{H}_{2} \mathrm{SO}_{4}$ overnight at $60{ }^{\circ} \mathrm{C}$. Finally, the black precipitate was washed to neutral with ultrapure water, obtaining the Co@NC catalyst.

\section{ASSOCIATED CONTENT}

\section{Supporting Information}

The Supporting Information is available free of charge at https://pubs.acs.org/doi/10.1021/acsomega.1c03427.

XRD patterns of the prepared ZIF-67 and simulated ZIF-67 (Figure S1); FTIR of ZIF-67 and 2-methylimidazole (Figure S2); SEM images of ZIF-67 (Figure S3); crystal structure diagram of ZIF-67 (Figure S4); TGA curve of ZIF-67 (Figure S5); and HTD peak integral area of pure AP and Co@NC/AP (Table S1) (PDF)

\section{AUTHOR INFORMATION}

\section{Corresponding Authors}

Qingchun Zhang - State Key Laboratory of Environmentfriendly Energy Materials, School of Materials Science and Engineering, Southwest University of Science and Technology, Mianyang 621010, P. R. China; Institute of Applied Physics 
and Materials Engineering, University of Macau, Macao SAR 999078, P. R. China; (1) orcid.org/0000-0003-4063-9645; Email: zhangqingchun@live.cn

Rufang Peng - State Key Laboratory of Environment-friendly Energy Materials, School of Materials Science and Engineering, Southwest University of Science and Technology, Mianyang 621010, P. R. China; Phone: +86-816-2419011; Email: pengrufang@swust.edu.cn

\section{Authors}

Huiyu Liu - State Key Laboratory of Environment-friendly Energy Materials, School of Materials Science and Engineering, Southwest University of Science and Technology, Mianyang 621010, P. R. China

Zhiliang Guo - State Key Laboratory of Environment-friendly Energy Materials, School of Materials Science and Engineering, Southwest University of Science and Technology, Mianyang 621010, P. R. China

Bo Jin - State Key Laboratory of Environment-friendly Energy Materials, School of Materials Science and Engineering, Southwest University of Science and Technology, Mianyang 621010, P. R. China; 이이이.org/0000-0002-6966-2876

Complete contact information is available at:

https://pubs.acs.org/10.1021/acsomega.1c03427

\section{Author Contributions}

H.L. and Z.G. contributed equally to this work; Q.Z. and B.J. conceived and designed the experiments; H.L. and Z.G. performed the experiments; H.L., Z.G., and R.P. analyzed the data; and Q.Z., Z.G., and H.L. wrote and edited the manuscript.

\section{Notes}

The authors declare no competing financial interest.

\section{ACKNOWLEDGMENTS}

The authors are grateful for financial support from the National Natural Science Foundation of China (Project No. 51972278), Sichuan Science and Technology Program (Project No. 2019YJ0447), the Project of State Key Laboratory of Environment-friendly Energy Materials, and the Southwest University of Science and Technology (Project No. 20fksy16).

\section{REFERENCES}

(1) Chaturvedi, S.; Dave, P. N. Solid propellants: AP/HTPB composite propellants. Arab. J. Chem. 2019, 12, 2061-2068.

(2) Dennis, C.; Bojko, B. On the combustion of heterogeneous AP/ HTPB composite propellants: A review. Fuel 2019, 254, No. 115646.

(3) Vyazovkin, S.; Wight, C. A. Kinetics of Thermal decomposition of cubic ammonium perchlorate. Chem. Mater. 1999, 11, 3386-3393.

(4) Marothiya, G.; Vijay, C.; Ishitha, K.; Ramakrishna, P. A. Effects on burn rates of pellets and propellants with catalyst-embedded AP. J. Propulsion Power 2018, 34, 969-974.

(5) Chaturvedi, S.; Dave, P. N.; Patel, N. N. Nano-Alloys: Potential catalyst for thermal decomposition of ammonium perchlorate. Synth. React. Inorg., Met.-Org., Nano-Met. Chem. 2014, 44, 258-262.

(6) Chaturvedi, S.; Dave, P. N. A review on the use of nanometals as catalysts for the thermal decomposition of ammonium perchlorate. $J$. Saudi Chem. Soc. 2013, 17, 135-149.

(7) Kapoor, I. P. S.; Srivastava, P.; Singh, G. Nanocrystalline transition metal oxides as catalysts in the thermal decomposition of ammonium perchlorate. Propellants Explos., Pyrotech. 2009, 34, 351356.
(8) Zhou, L.; Cao, S.; Zhang, L.; Xiang, G.; Wang, J.; Zeng, X.; Chen, J. Facet effect of $\mathrm{Co} 3 \mathrm{O} 4$ nanocatalysts on the catalytic decomposition of ammonium perchlorate. J. Hazard. Mater. 2020, 392, No. 122358

(9) Chaturvedi, S.; Dave, P. N. Nano-metal oxide: potential catalyst on thermal decomposition of ammonium perchlorate. J. Exp. Nanosci. 2012, 7, 205-231.

(10) Hosseini, S. G.; Khodadadipoor, Z.; Mahyari, M. CuO nanoparticles supported on three-dimensional nitrogen-doped graphene as a promising catalyst for thermal decomposition of ammonium perchlorate. Appl. Organomet. Chem. 2018, 32, No. e3959.

(11) Zhang, Y.; Li, K.; Liao, J.; Wei, X.; Zhang, L. Microwaveassisted synthesis of graphitic carbon nitride $/ \mathrm{CuO}$ nanocomposites and the enhancement of catalytic activities in the thermal decomposition of ammonium perchlorate. Appl. Surf. Sci. 2020, 499, No. 143875.

(12) Zhou, Q.; Jin, B.; Chen, J. J.; Xiao, Y. Y.; Chu, S. J.; Peng, R. F. Facile fabrication of $\mathrm{Cu}$-doped carbon aerogels as catalysts for the thermal decomposition of ammonium perchlorate. Appl. Organomet. Chem. 2020, 34, No. e5700.

(13) Wang, X. B.; Li, J. Q.; Luo, Y. J. Effect of drying methods on the structure and thermal decomposition behavior of ammonium perchlorate/graphene composites. Acta. Phys.-Chim. Sin. 2013, 10, 2079-2086.

(14) Cui, P.; Li, F. S.; Zhou, J.; Jiang, W.; Yang, Y. Preparation and thermal decomposition behavior of carbon nanotubes/ammonium perchlorate composite particles. Chin. J. Explos. Propellants 2006, 29, $25-28$.

(15) Zhang, W. W.; Luo, Q. P.; Duan, X. H.; Zhou, Y.; Pei, C. H. Nitrated graphene oxide and its catalytic activity in thermal decomposition of ammonium perchlorate. Mater. Res. Bull. 2014, 50, 73-78.

(16) Abdelhafiz, M.; Yehia, M.; Mostafa, H. E.; Wafy, T. Z. Catalytic action of carbon nanotubes on ammonium perchlorate thermal behavior. React. Kinet. Mech. Catal. 2020, 131, 353-366.

(17) Chen, J.; He, S. M.; Huang, B.; Zhang, L. Y.; Qiao, Z. Q.; Wang, J.; Yang, G. C.; Huang, H.; Hao, Q. L. Highly space-confined ammonium perchlorate in three-dimensional hierarchically ordered porous carbon with improved thermal decomposition properties. Appl. Surf. Sci. 2018, 457, 508-515.

(18) Jiao, L.; Jiang, H. L. Metal-organic-framework-based singleatom catalysts for energy applications. Chem 2019, 5, 786-804.

(19) Huang, H. G.; Shen, K.; Chen, F. F.; Li, Y. W. Metal-organic frameworks as a good platform for the fabrication of single-atom catalysts. ACS Catal. 2020, 10, 6579-6586.

(20) Song, Z.; Zhang, L.; Doyle-Davis, K.; Fu, X.; Sun, X.; et al. Recent Advances in MOF-derived single atom catalysts for electrochemical applications. Adv. Energy Mater. 2020, 10, No. 2001561.

(21) Duan, H.; Lin, X.; Liu, G.; Xu, L.; Li, F. S. Synthesis of Co nanoparticles and their catalytic effect on the decomposition of ammonium perchlorate. Chin. J. Chem. Eng. 2008, 16, 325-328.

(22) Hosseini, S. G.; Toloti, S. J. H.; Babaei, K.; Ghavi, A. The effect of average particle size of nano- $\mathrm{Co}_{3} \mathrm{O}_{4}$ on the catalytic thermal decomposition of ammonium perchlorate particles. J. Therm. Anal. Calorim. 2016, 124, 1243-1254.

(23) Zhao, Y.; Zhang, X.; Xu, X.; Zhao, Y.; Zhou, H.; Li, J.; Jin, H. The synthesis of ultra-long cobalt chains and its outstanding catalytic performance on the thermal decomposition of ammonium perchlorate. Mater. Chem. Phys. 2017, 201, 235-240.

(24) Jiang, X. L.; Li, H. B.; Xiao, J. P.; Gao, D. F.; Si, R.; Yang, F.; Li, Y. S.; Wang, G. X.; Bao, X. H. Carbon dioxide electroreduction over imidazolate ligands coordinated with $\mathrm{Zn}(\mathrm{II})$ center in ZIFs. Nano Energy 2018, 52, 345-350.

(25) Li, X. Y.; Gao, X. Y.; Ai, L. H.; Jiang, J. Mechanistic insight into the interaction and adsorption of $\mathrm{Cr}(\mathrm{VI})$ with zeolitic imidazolate framework-67 microcrystals from aqueous solution. Chem. Eng. J. 2015, 274, 238-246.

(26) Zhou, K.; Mousavi, B.; Luo, Z. X.; Phatanasri, S.; Chaemchuen, S.; Verpoort, F. Characterization and properties of $\mathrm{Zn} / \mathrm{Co}$ zeolitic 
imidazolate frameworks vs. ZIF-8 and ZIF-67. J. Mater. Chem. A 2017, $5,952-957$

(27) Hai, X.; Zhao, X. X.; Guo, N.; Yao, C. H.; Chen, C.; Liu, W.; Du, Y. H.; Yan, H.; Li, J.; Chen, Z. X.; Li, X.; Li, Z. J.; Xu, H.; Lyu, P.; Zhang, J.; Lin, M.; Su, C. L.; Pennycook, S. J.; Zhang, C.; Xi, S. B.; Lu, $\mathrm{J}$. Engineering local and global structures of single Co atoms for a superior oxygen reduction reaction. ACS Catal. 2020, 10, 5862-5870.

(28) Tang, J.; Wang, T.; Pan, X. C.; Sun, X.; Fan, X. L.; Guo, Y. X.; Xue, H. R.; He, J. P. Synthesis and electrochemical characterization of $\mathrm{N}$-Doped partially graphitized ordered mesoporous carbon-Co composite. J. Phys. Chem. C 2013, 117, 16896-16906.

(29) Tang, J.; Salunkhe, R. R.; Liu, J.; Torad, N. L.; Imura, M.; Furukawa, S.; Yamauchi, Y. Thermal conversion of core-shell metalorganic frameworks: A new method for selectively functionalized nanoporous hybrid carbon. J. Am. Chem. Soc. 2015, 137, 1572-1580.

(30) Xia, Y.; Mokaya, R. Generalized and facile synthesis approach to $\mathrm{N}$-doped highly graphitic mesoporous carbon materials. Chem. Mater. 2005, 17, 1553-1560.

(31) Zhao, C. M.; Dai, X. Y.; Yao, T.; Chen, W. X.; Wang, X. Q.; Wang, J.; Yang, J.; Wei, S. Q.; Wu, Y.; Li, Y. D. Ionic exchange of metal-organic frameworks to access single nickel sites for efficient electroreduction of $\mathrm{CO}_{2}$. J. Am. Chem. Soc. 2017, 139, 8078-8081.

(32) Zhang, W.; Jiang, X.; Wang, X.; Kaneti, Y. V.; Chen, Y.; Liu, J.; Jiang, J. S.; Yamauchi, Y.; Hu, M. Spontaneous weaving of graphitic carbon networks synthesized by pyrolysis of ZIF-67 crystals. Angew. Chem., Int. Ed. 2017, 56, 8435-8440.

(33) Biesinger, M. C.; Payne, B. P.; Grosvenor, A. P.; Lau, L.; Gerson, A. R.; Smart, R. Resolving surface chemical states in XPS analysis of first row transition metals, oxides and hydroxides: $\mathrm{Cr}, \mathrm{Mn}$, Fe, Co and Ni. Appl. Surf. Sci. 2011, 257, 2717-2730.

(34) Tripathy, S. K.; M Christy, M.; Park, N. H.; Suh, E. K.; Anand, S.; Yu, Y. T. Hydrothermal synthesis of single-crystalline nanocubes of $\mathrm{Co}_{3} \mathrm{O}_{4}$. Mater. Lett. 2008, 62, 1006-1009.

(35) Mou, J.; Li, Y.; Liu, T.; Zhang, W.; Li, M.; Xu, Y.; Zhong, L.; Pan, W.; Yang, C.; Huang, J.; Liu, M. Metal-organic frameworksderived nitrogen-doped porous carbon nanocubes with embedded Co nanoparticles as efficient sulfur immobilizers for room temperature sodium-sulfur batteries. Small Methods 2021, 5, No. 2100455.

(36) Rosser, W. A.; Inami, S. H.; Wise, H. Thermal decomposition of ammonium perchlorate. Combust. Flame 1968, 12, 427-435.

(37) Reid, D. L.; Russo, A. E.; Carro, R. V.; Stephens, M. A.; Lepage, A. R.; Spalding, T. C.; Petersen, E. L.; Seal, S. Nanoscale additives tailor energetic materials. Nano Lett. 2007, 7, 2157-2161.

(38) Tang, G.; Tian, S.; Zhou, Z.; Wen, Y.; Pang, A.; Zhang, Y.; Zeng, D.; Li, H.; Shan, B.; Xie, C. ZnO micro/nanocrystals with tunable exposed (0001) facets for enhanced catalytic activity on the thermal decomposition of ammonium perchlorate. J. Phys. Chem. C 2014, 118, 11833-11841.

(39) Zheng, Z. L.; Zhang, W. C.; Chen, L.; Xiong, W. H.; Zeng, G. W.; Liu, J. Q.; Wu, R. H.; Wang, J. X.; Ye, J. H.; Zhu, J. W. In-situ synthesis of $\mathrm{MnCo}_{2} \mathrm{O}_{4.5}$ nanosheets on reduced graphene oxide for a great promotion in the thermal decomposition of ammonium perchlorate. Appl. Surf. Sci. 2019, 483, 496-505.

(40) Ayoman, E.; Hosseini, S. G. Synthesis of $\mathrm{CuO}$ nanopowders by high-energy ball-milling method and investigation of their catalytic activity on thermal decomposition of ammonium perchlorate particles. J. Therm. Anal. Calorim. 2016, 123, 1213-1224.

(41) Hosseini, S. G.; Ayoman, E. Synthesis of alpha- $\mathrm{Fe}_{2} \mathrm{O}_{3}$ nanoparticles by dry high-energy ball-milling method and investigation of their catalytic activity. J. Therm. Anal. Calorim. 2017, 128, 915-924.

(42) An, T.; He, W.; Chen, S. W.; Zuo, B. L.; Qi, X. F.; Zhao, F. Q.; Luo, Y.; Yan, Q. L. Thermal behavior and thermolysis mechanisms of ammonium perchlorate under the effects of graphene oxide-doped complexes of triaminoguanidine. J. Phys. Chem. C 2018, 122, 2695626964.

(43) Han, X.; Zhou, L.; Cao, S.; Zhang, L.; Xiang, G.; Chen, J. F. Exploring the roles of ZIF-67 as an energetic additive in the thermal decomposition of ammonium perchlorate. Energy Fuels 2021, 35, $4447-4456$

(44) Guo, Z.; Zhang, Q.; Liu, H.; Zhang, H.; Peng, R. A novel metalorganic framework precursor strategy to fabricate sub-micron $\mathrm{CuO}$ microspheres for catalytic thermal decomposition of ammonium perchlorate. Mater. Today Commun. 2021, 26, No. 102139.

(45) Chen, J.; He, S.; Bing, H.; Zhang, L.; Qiao, Z.; Wang, J.; Yang, G.; Huang, H.; Hao, Q. Highly space-confined ammonium perchlorate in three-dimensional hierarchically ordered porous carbon with improved thermal decomposition properties. Appl. Surf. Sci. 2018, 457, 508-515.

(46) Zhou, L. Y.; Cao, S. B.; Zhang, L. L.; Xiang, G. L.; Wang, J. X.; Zeng, X. F.; Chen, J. F. Facet effect of $\mathrm{Co}_{3} \mathrm{O}_{4}$ nanocatalysts on the catalytic decomposition of ammonium perchlorate. J. Hazard. Mater. 2020, 392, No. 122358.

(47) Wight, C. A.; Vyazovkin, S. Kinetics of thermal decomposition of cubic ammonium perchlorate. Chem. Mater. 1999, 11, 3386-3393.

(48) Li, L. P.; Sun, X. F.; Qiu, X. Q.; Xu, J. X.; Li, G. S. Nature of catalytic activities of $\mathrm{CoO}$ nanocrystals in thermal decomposition of ammonium perchlorate. Inorg. Chem. 2008, 47, 8839-8846. 